\title{
First- $v$. second-generation antipsychotics and risk for diabetes in schizophrenia: systematic review and meta-analysis
}

\author{
M. Smith, D. Hopkins, R. C. Peveler, R. I. G. Holt, M. Woodward and K. Ismail
}

\section{Background}

The increased prevalence of diabetes in schizophrenia is partly attributed to antipsychotic treatment, in particular second-generation antipsychotics, but the evidence has not been systematically reviewed.

\begin{abstract}
Aims
Systematic review and meta-analysis comparing diabetes risk for different antipsychotics in people with schizophrenia.
\end{abstract}

\section{Method}

We searched MEDLINE, PSycINFO, EMBASE, International Pharmaceutical Abstracts, CINAHL and Web of Knowledge until September 2006. Studies were eligible for inclusion if the design was cross-sectional, case-control, cohort or a controlled trial in individuals with schizophrenia or related psychotic disorders, where second-generation antipsychotics (defined as clozapine, olanzapine, risperidone and quetiapine) were compared with first-generation antipsychotics and diabetes was an outcome. Data were pooled using random effects inverse variance weighted meta-analysis.

\section{Results}

Of the studies that met the inclusion criteria $(n=14), 11$ had sufficient data to include in the meta-analysis. Four of these were retrospective cohort studies. The relative risk of diabetes in patients with schizophrenia prescribed one of the second-generation $v$. first-generation antipsychotics was 1.32 (95\% Cl 1.15-1.51). There were insufficient data to include aripiprazole, ziprasidone and amisulpride in this analysis.

\section{Conclusions}

There is tentative evidence that the second-generation antipsychotics included in this study are associated with a small increased risk for diabetes compared with firstgeneration antipsychotics in people with schizophrenia. Methodological limitations were found in most studies, leading to heterogeneity and difficulty interpreting data. Regardless of type of antipsychotic, screening for diabetes in all people with schizophrenia should be routine.

\section{Declaration of interest}

R.P. has received fees for speaking and consulting from makers of antipsychotics, including Eli Lilly and Company, Bristol Myers Squibb, Sanofi, Pfizer, Janssen and Astra Zeneca. R.H. has received educational grants and fees for lecturing and consultancy work from Eli Lilly and Company, Bristol Myers Squibb and GlaxoSmithKline.
Diabetes mellitus is characterised by chronic hyperglycaemia and is one of the most common chronic diseases. The prevalence of detected diabetes is around $3-4 \%$ in the general population ${ }^{1}$ and set to double worldwide by $2030,{ }^{2}$ largely as a result of an ageing population and the epidemic of obesity. ${ }^{3}$ The most common types of diabetes are types 1 and 2. Type 1 diabetes represents around $10-15 \%$ of all cases of diabetes and is associated with absolute insulin deficiency. Type 2 diabetes accounts for $85-90 \%$ of all cases and results from a combination of insulin resistance and relative insulin deficiency.

The prevalence of diabetes has been reported to be two- to threefold higher in people with schizophrenia than the general population. ${ }^{4-8}$ The reason for this increase is not well understood but likely to be multifactorial. ${ }^{9}$

There has been increasing concern that antipsychotics, especially the second-generation type, are also associated with an increased risk for diabetes in people with schizophrenia. ${ }^{10-12}$ Despite this increase in case reports, commentaries and editorials, there has been no systematic review and meta-analysis of the literature. Establishing the evidence base for this apparent phenomenon which is of increasing concern to psychiatrists and people with schizophrenia is crucial prior to developing guidelines for diabetes screening and management.

We conducted a systematic review of the evidence for an association between diabetes and type of antipsychotic medication, and carried out a meta-analysis of the relative risk of diabetes in people with schizophrenia who were prescribed second-generation antipsychotics, compared with those who were prescribed firstgeneration antipsychotics.

\section{Methods}

\section{Criteria for selecting studies}

Abstracts were considered eligible for full manuscript data extraction if they fulfilled the following criteria:

(a) the design was a cross-sectional, case-control, cohort or controlled trial

(b) the study population included children or adults with schizophrenia or related psychotic disorders

(c) second-generation antipsychotics (defined in this study as clozapine, olanzapine, risperidone and quetiapine) were being compared with first-generation antipsychotics (listed in the British National Formulary (BNF) $)^{13}$

(d) measurement of diabetes as a primary or secondary outcome was included.

Studies were excluded according to the criteria shown in Fig. 1. Studies funded by pharmaceutical companies were included, and coded as such, whether fully or partially funded.

\section{Search strategy}

Using established Cochrane Collaboration search strategies and definitions for schizophrenia, antipsychotics and diabetes mellitus, we searched the following electronic libraries: MEDLINE (1966 to September 2006); PsycINFO (1967 to September 2006); EMBASE (1980 to September 2006); International Pharmaceutical Abstracts 


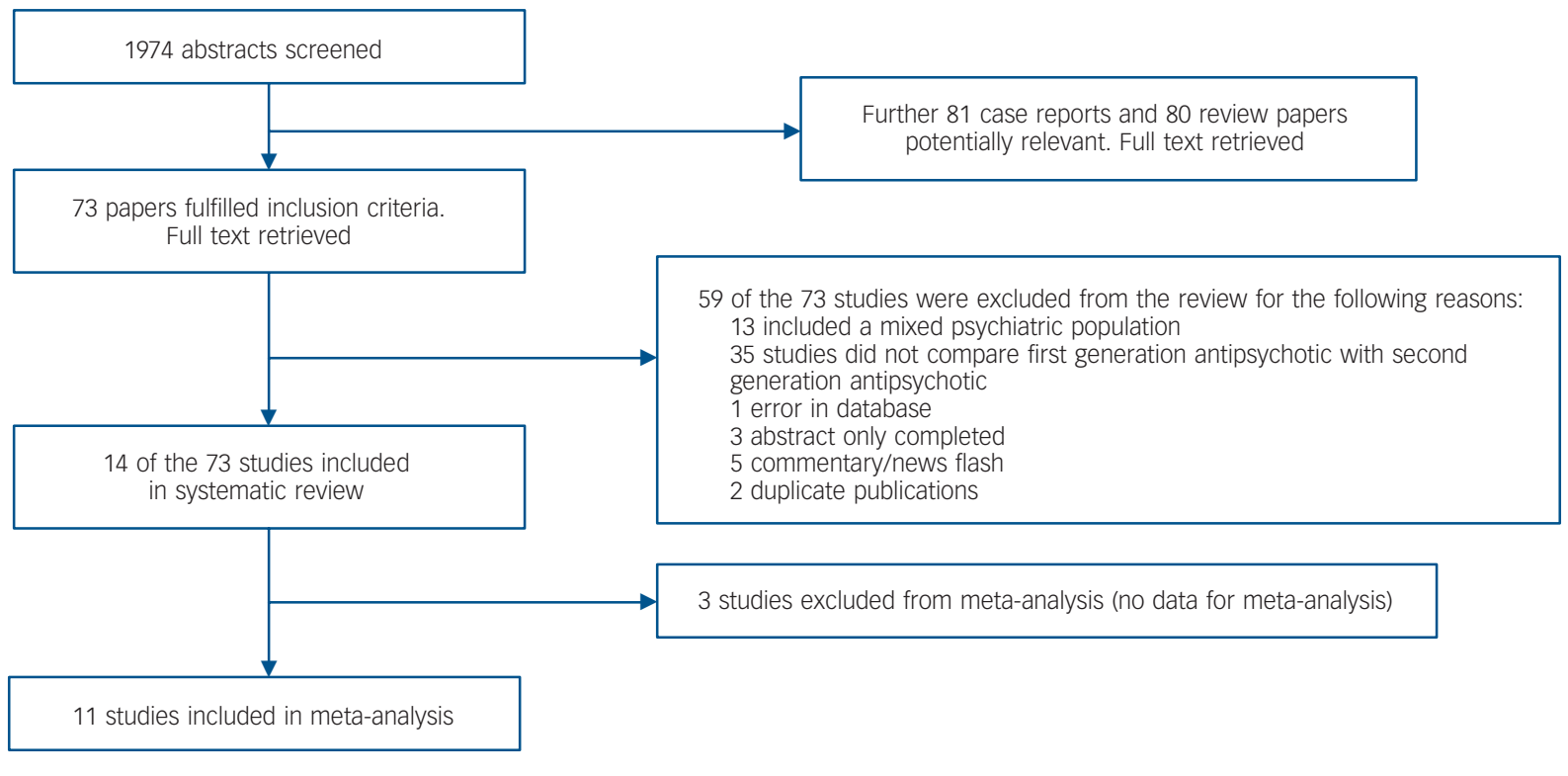

Fig. 1 Flow chart of systematic review.

(1970 to September 2006); CINAHL Cumulative Index to Nursing and Allied Health Literature (1982 to September 2006); and Web of Knowledge (1981 to September 2006). The following search terms were used: 'schizophrenia and related illnesses', adapted from the Cochrane Schizophrenia Group schizophrenia search strategy; 'antipsychotic', including all first-generation antipsychotics in the BNF; ${ }^{13}$ 'diabetes mellitus', amended from the Cochrane Collaboration Metabolic and Endocrine Disorder Group general diabetes search strategy (search strategy available from authors on request).

The proceedings of conferences during 2000-2005 on diabetes (American Diabetes Association, Diabetes UK, European Association for the Study of Diabetes, International Diabetes Federation) and psychiatry (American Psychiatric Association, Schizophrenia Research) were hand and electronically searched in the relevant sections.

The reference lists of included studies and reviews were searched for any additional studies. Although clozapine was first introduced in 1956 and withdrawn shortly after because of severe side-effects, there was a small risk of not detecting pre-1966 studies (when MEDLINE began), which was addressed by checking reference lists.

Corresponding authors and experts in the field were contacted for additional information on published and unpublished studies.

\section{Data extraction}

The abstracts of studies identified by electronic searches were examined by M.S. If unclear, the original article was retrieved, reviewed and discussed with co-investigators.

Data were extracted from papers selected for further review. Foreign papers were translated by doctors who were native speakers in that language.

Using a standardised data extraction sheet, we recorded and coded the following information (if available) from studies: country of origin; study design; setting; total sample size (and total number taking second-generation and first-generation antipsychotics); classification and method of assessment of schizophrenia; ascertainment of antipsychotic exposures; assessment and classification of outcome (diabetes); follow-up period; age; type of confounders adjusted; and source of funding. An attempt to retrieve missing data in the published article was made by contacting the author for correspondence on at least two occasions by email or letter.

\section{Quality assessment}

There is no consensus standardised method for the quality assessment of observational studies. We adapted the Meta-analysis of Observational Studies in Epidemiology (MOOSE) ${ }^{14}$ and Strengthening of the Reporting of Observational Studies in Epidemiology (STROBE) ${ }^{15}$ guidelines to assess and code the quality of the studies.

A study method was considered as high-quality if the following criteria were present:

(a) the design was prospective, including randomised controlled trials (RCTs)

(b) case definition for participants with schizophrenia was based on the past three editions of the ICD or the DSM

(c) the study included only patients who did not have diabetes at recruitment (baseline) of the sample

(d) recruitment of the sample was consecutive or random

(e) ascertainment of antipsychotic medication exposure used an objective technique, such as screening of electronic prescription databases

(f) ascertainment of diabetes mellitus as an outcome was based on accepted diagnostic criteria, such as the American Diabetes Association $(\mathrm{ADA})^{16}$ and the World Health Organization $(\mathrm{WHO})^{17}$

(g) temporality of an association between antipsychotic and diabetes was assessed with at least 1 year of follow-up reported

(h) the data were controlled for the six main independent risk factors for diabetes (body mass index, family history of diabetes (first-degree relatives), ethnicity, age, physical activity and socio-economic status).

All other descriptions, or lack of any description, were coded as low-quality. 
Setting and funding status of the studies were recorded but not coded for quality as it was difficult to assess with confidence how this could have introduced a bias.

We did not summarise the quality of studies with a score as this approach has been criticised as allocating equal weight to different aspects of methodology. ${ }^{18}$

\section{Statistical analysis}

Quantitative data from studies of high quality were pooled using random effects inverse variance weighted meta-analysis ${ }^{19}$ in Stata, version 9 for Windows. Where available, approximate relative risks (odds ratios or hazard ratios) and corresponding 95\% confidence intervals (CIs) for diabetes, which compared second-generation antipsychotics with first-generation antipsychotics, were used from each study. Where results were given after different adjustments for confounding factors, the result for the largest confounding set was used. If only numbers with diabetes by drug type were available, these were first used to compute unadjusted odds ratios and corresponding 95\% CIs for that study. Where no diabetes events occurred in any drug group, the study was excluded from the meta-analysis. Some studies gave separate results for different second-generation drugs but no overall result. In these cases, meta-analysis was used to create a pooled result, over all second-generation drugs, for that study only. This result was carried forward to the overall meta-analysis. The percentage of heterogeneity between studies not attributable to random noise was estimated using Higgins' I-squared statistic. $^{19}$

\section{Results}

The search strategy identified 1974 abstracts from which 73 full texts were selected for further extraction. Data extraction from full texts identified 14 studies that met the criteria for inclusion in the systematic review. A total of 11 studies had sufficient information to be included in the meta-analysis. The number of studies that were excluded from the review and meta-analysis are shown in Fig. 1. The studies included in the systematic review are described in the online Table DS1.

Of the 14 studies, 10 were based in a hospital setting, 1 was based in primary care and 3 comprised a mixed hospital and primary care setting. Seven studies used a health administration or health insurance database. ${ }^{8,20-25}$ Only 1 study used a database designed for conducting research. ${ }^{26}$

The majority of study designs were either cross-sectional $(n=4)$, retrospective cohort $(n=4)$ or case-control $(n=2)$ with only a handful using prospective design $(n=3)$. The majority used consecutive or convenience sample selection, with two studies using random selection (Table DS1).

Nine (64.3\%) studies used a classification system for schizophrenia (ICD, DSM-IV) but the majority were based on a clinical assessment with only one study using a research diagnostic interview, namely the Structured Clinical Interview for DSM-III- $\mathrm{R}^{27}$ (Table DS1). The average age was 45.0 years (s.d.=7.7) $(n=12$ studies with age data).

Second-generation antipsychotics included in these studies were clozapine, olanzapine, risperidone and quetiapine, with clozapine studied on its own in four of the studies. ${ }^{5,23,28,29}$ Although sulpiride is classified as a second-generation antipsychotic in one paper, ${ }^{30}$ it is classified as a first-generation in the BNF and therefore was excluded from our list of secondgeneration antipsychotics.

The diagnosis of diabetes mellitus was according to $\mathrm{WHO}^{31}$ guidelines in $3(21.4 \%)$ studies, ${ }^{5,28,30}$ ICD-9 diagnosis in 5
(35.7\%) studies, ${ }^{8,20,21,23,25}$ and ADA guidelines in $3(21.4 \%)$ studies. ${ }^{29,32,33}$ Most of the studies ensured that there was no evidence of diabetes prior to antipsychotic use but certain studies did not clearly differentiate between new and existing cases of diabetes. ${ }^{8,29,30}$ In addition, some studies used prescription of diabetes medication for diagnosis of diabetes. ${ }^{20,21,23-26}$

Excluding cross-sectional studies, follow-up periods ranged from 2 to 62 months and the median duration of follow-up from the start of the study was 12 months (interquartile range 2.6-16.8 months).

Most studies did not adjust for confounders in the methods or analysis. Studies also did not adjust for all risk factors for diabetes (Table DS1).

There were 7 studies funded by the pharmaceutical industry. ${ }^{20-22,24-26,33}$ One study was translated into English. ${ }^{32}$

Our meta-analysis found an overall relative risk of a diagnosis of diabetes in people with schizophrenia prescribed a secondgeneration antipsychotic of 1.32 (95\% CI 1.15-1.51) compared with being prescribed a first-generation antipsychotic (Fig. 2). The percentage of variability across studies that is attributable to heterogeneity rather than chance was $80 \%$ (95\% CI 66-89) and the test for heterogeneity was highly significant $(P<0.001)$. Both these results suggest that there is considerable variation between the studies.

To explain this heterogeneity, sensitivity analyses were carried out. Relative risks for separate second-generation drugs were as follows: risperidone 1.16 (95\% CI $0.99-1.35$; $n=6$ studies), quetiapine 1.28 (95\% CI 1.14-1.45; $n=3$ studies), olanzapine 1.28 (95\% CI 1.12-1.45; $n=8$ studies) and clozapine 1.39 (95\% CI 1.24-1.55; $n=7$ studies).

\section{Discussion}

The study criteria for this systematic review identified 14 papers comparing the risk of having or developing diabetes while on second generation antipsychotics with first-generation antipsychotics in people with schizophrenia or related disorders. Eleven of these studies had sufficient data to pool. Meta-analysis found that second-generation antipsychotics were associated with a small increased relative risk for diabetes compared with firstgeneration antipsychotics. ${ }^{34}$ Methodological limitations in nearly all the studies were evidenced by significant heterogeneity and this raises some difficulties in interpreting and comparing the studies.

\section{Strengths and limitations}

The strengths of our review are that the study is based on principles derived from the Cochrane Collaboration methods, such as an a priori protocol. In addition, established Cochrane Collaboration search strategies were adapted as well as hand searching of references of relevant papers, including non-English ones. Experts in the field were contacted for missing data and further information. We also conducted separate analyses comparing individual second-generation antipsychotics with first-generation antipsychotics to take account of the unequal distribution and potentially different diabetogenic potential of individual drugs.

A possible limitation of our review is that we used a narrow search strategy which may have excluded well-designed studies that focused on related outcomes other than diabetes, such as weight gain or insulin levels. Although cardiovascular risk factors other than diabetes mellitus such as abnormal lipid metabolism, blood pressure and obesity may also have a significant effect on the physical health of patients with schizophrenia, we chose to focus on diabetes and exclude metabolic syndrome. Although the diagnosis of diabetes is relatively uniform, no such consensus 


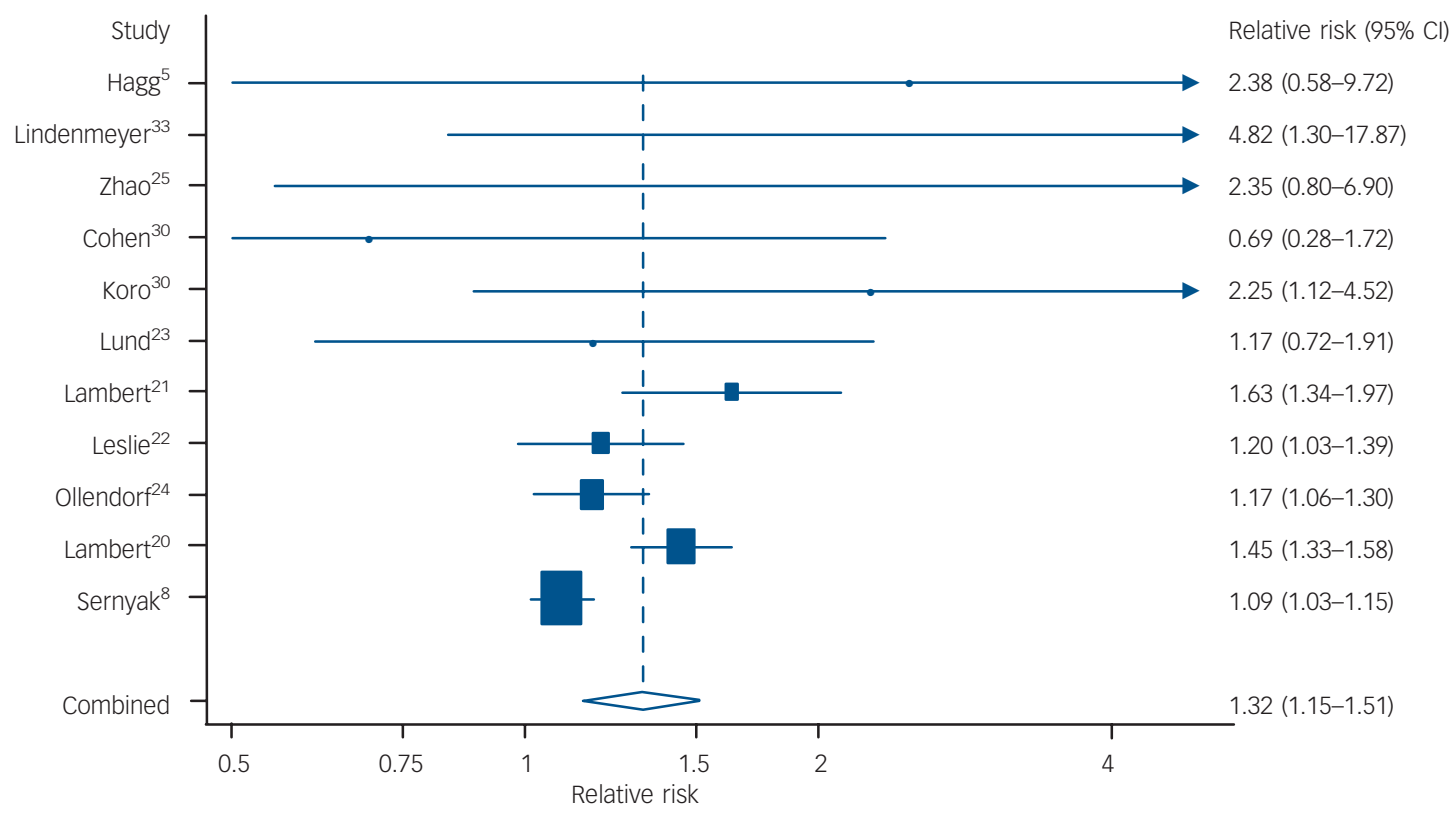

Fig. 2 Forest plot of relative risks and $95 \% \mathrm{Cls}$ for diabetes in patients on first-generation antipsychotics compared with secondgeneration antipsychotics.

Estimates are at the centre of the boxes, which are drawn in proportion to the standard errors; lines show $95 \%$ Cls. Arrows denote censoring. The diamond shows the combined (pooled) estimate at its centre; its horizontal points lie at the $95 \%$ confidence limits for the combined estimate.

exists for metabolic syndrome. ${ }^{9}$ We also chose to exclude homoeostasis model assessment as an outcome as it is a research tool and its validity for predicting diabetes remains unclear. We excluded patients taking antipsychotics when the clinical indication was not clear. ${ }^{35}$ We excluded studies of mixed psychiatric populations where subgroups of patients with schizophrenia who developed diabetes could not be distinguished. ${ }^{36-41}$

This led to some studies that included people with schizophrenia being excluded. The rationale for including only schizophrenia and related illnesses, and excluding all other psychiatric illnesses or studies which did not state diagnosis, was the importance of considering schizophrenia as an independent risk factor for diabetes. Studies that did not compare a secondgeneration antipsychotic with a first-generation antipsychotic, but instead to another second-generation antipsychotic or placebo were excluded. ${ }^{42-45}$ We included all second-generation antipsychotics but there were no studies that met our eligibility criteria for the more recent drugs such as ziprasidone, amisulpride and aripiprazole, which have been suggested to be less diabetogenic in some case reports. ${ }^{46,47}$ Future studies comparing their diabetogenic profiles are awaited.

\section{Quality of studies}

We found that the average duration of studies was around 12 months with three studies having a follow up of only 3 months or less. It could be that these studies did not follow up their patients for long enough, perhaps several years, to capture the long-term risk for diabetes and this may have led to an underestimation of our findings. However, there is also some evidence that the diabetogenic effects are rapid in onset - within the first few months. ${ }^{48}$ It is possible that there may be several glucosemetabolism-altering mechanisms, a short- and a long-term process, and studies with repeated measurements over several years could help to elucidate the diabetogenic patterns.
Most studies included in this review were retrospective or pharmacoepidemiological using large databases. Limitations in analysing data from such studies, mainly due to the quality of clinical data recorded, exist. Database studies do not rely on a standardised method of diagnosing diabetes mellitus. Random blood glucose measures were often used, highlighting the difficulty of obtaining fasting blood samples from this patient group. Furthermore, utilisation of established diagnostic guidelines for diabetes such as those provided by the $\mathrm{WHO}^{17}$ or $\mathrm{ADA}^{16}$ was inconsistent. Prescription of hypoglycaemic medication as a method of diagnosis may exclude patients receiving nonpharmacological treatment for diabetes such as those on diet alone. It may also include some people receiving metformin for other indications such as polycystic ovarian syndrome. Little evidence existed that the diagnosis of diabetes in databases was reliable or valid. The date of the first listed claim may not represent the actual date of onset of diabetes as the diagnosis may have been previously made but recorded elsewhere.

The techniques and methods used to diagnose schizophrenia cannot be inferred accurately from the databases. We observed that not all antipsychotics were included in the databases. This may be a reflection of the country where the study was undertaken and the availability of, and preference for, one type of antipsychotic over another. Many of the databases were of specific populations and mainly in the USA, which may limit their generalisability to other populations. In addition, a number of studies had only a few weeks or months of observation which may have captured those individuals who have rapid weight gain but which may be too short a time to identify later onset of diabetes.

\section{Association between antipsychotics and diabetes}

The first observation of an association between antipsychotics and diabetes occurred over 50 years ago, but renewed interest in this 
link has developed with second-generation antipsychotics in the past decade. Second-generation antipsychotics have been shown to reduce extrapyramidal side-effects and are beneficial to many patients. ${ }^{49}$ However, the concerns that second-generation antipsychotics may also increase the risk of metabolic dysregulation must also be carefully considered.

As the majority of studies were cross-sectional or retrospective cohort in design, it is not possible to determine the direction of the association between second-generation antipsychotics and diabetes. Our review highlights that most studies were limited in quality with none of the studies fulfilling all of the quality criteria; five studies had four or fewer quality criteria (Table DS1). Any association detected in this review is likely to be significantly biased. The possibility of residual confounding also cannot be dismissed. One residual confounder not reported in any of the studies was whether an increased amount of screening occurred in those on second-generation antipsychotics compared with firstgeneration antipsychotics, owing to greater clinical awareness. This is important because of the high prevalence of undiagnosed diabetes in people with schizophrenia, among whom as many as $75 \%$ of cases of diabetes may be missed. ${ }^{50}$

Our review found that second-generation antipsychotics were associated with a $30 \%$ increased risk of diabetes compared with first-generation antipsychotics in people with schizophrenia - this is probably a biased observation ${ }^{33,51}$ with the true association probably being smaller. This review was unable to find sufficient evidence to differentiate the risk associated with individual antipsychotics, nor did the studies we identified help shed any light on causal mechanisms. Our review emphasises that, to date, the evidence is very poor and should not be used alone as a guideline for or switching antipsychotic medications or implementing diabetes screening and management protocols for schizophrenia until further evidence comes to light.

In epidemiological terms, a relative risk of less than 2 is considered low, whereas a relative risk of greater than 3 is considered high. ${ }^{51}$ This is an important clinical issue to consider when treating patients with antipsychotic medication. Importantly, clinicians should implement protocols for identifying physical illnesses, in particular diabetes, in people with schizophrenic illnesses, while also reviewing the rationale and dosage of prescribing antipsychotic medication both in terms of treatment of psychotic symptoms and risk of diabetes. Our review has identified the key confounders and methodological issues that need to be considered in evaluating the association between diabetes and antipsychotic medication in schizophrenia. There also remains an urgent need for continuing to identify and understand the biological pathways and increase the epidemiological evidence base for diabetes in schizophrenia. In particular, randomised controlled trials comparing first- and second-generation drugs should consider the implications of their study's power to detect differences in onset of diabetes as an adverse event, bearing in mind these events are likely to be rare.

\footnotetext{
M. Smith, MRCPsych, Department of Psychological Medicine, Institute of Psychiatry, London, UK; D. Hopkins, FRCP, Department of Diabetic Medicine, King's College Hospital, London, UK; R. C. Peveler, MA, DPhil, FRCPsych, Clinical Neurosciences Division, University of Southampton, Southampton, UK; R. I. G. Holt, FRCP, FHEA, $\mathrm{PhD}$, Endocrinology and Metabolism Sub-division, University of Southampton, Southampton, UK; M. Woodward, PhD, Department of Medicine, Mount Sinai Medical Center, New York, USA; K. Ismail, PhD, MRCPsych, Department of Psychological Medicine, Institute of Psychiatry, King's College London, UK

Correspondence: M. Smith, Department of Psychological Medicine, Institute of Psychiatry, King's College London, London SE5 9RJ, UK. Email: m.smith@iop.kcl.ac.uk

First received 20 Feb 2007, final revision 21 Jan 2008, accepted 13 Feb 2008
}

\section{Acknowledgements}

There was no funding for this study. We would like to thank Hugh McGuire (Institute of Psychiatry, King's College London) and Dr Alex Mitchell (Leicester) respectively for help with the search strategy, and Dr Zerrin Atakan (Institute of Psychiatry, King's College London), Dr Carmine Pariante (Institute of Psychiatry, King's College London), and Dr Smith for translation of articles written in Turkish, Italian and Mandarin respectively.

\section{References}

1 International Diabetes Federation. Diabetes Atlas. International Diabetes Federation, 2003.

2 Wild S, Roglic G, Green A, sicree R, King H. Global prevalence of diabetes: estimates for the year 2000 and projections for 2030. Diabetes Care 2004; 27: 1047-53.

3 Amos A, McCarty D, Zimmet P. The rising global burden of diabetes and its complications: estimates and projections to the year 2010. Diabet Med 1997; 14: S7-85.

4 Dixon L, Weiden P, Delahanty J. Prevalence and correlates of diabetes in national schizophrenia samples. Schizophr Bull 2000; 2: 903-12.

5 Hägg S, Joelsson L, Mjörndal T, Spigset O, Oja G, Dahlqvist R. Prevalence of diabetes and impaired glucose tolerance in patients treated with clozapine compared with patients treated with conventional depot neuroleptic medications. J Clin Psychiatry 1998; 59: 294-9.

6 Mukherjee S, Decina P, Bocola V, Saraceni F, Scapicchio PL. Diabetes mellitus in schizophrenic patients. Compr Psychiatry 1996; 37: 68-73.

7 Regenold WT, Thapar RK, Marano C, Gavirneni S, Kondapavuluru PV. Increased prevalence of type 2 diabetes mellitus among psychiatric inpatients with bipolar I affective and schizoaffective disorders independent of psychotropic drug use. J Affective Disord 2002; 70: 19-26.

8 Sernyak MJ, Leslie DL, Alarcon RD, Losonczy MF, Rosenheck R. Association of diabetes mellitus with use of first generation neuroleptics in the treatment of schizophrenia. Am J Psychiatry 2002; 159: 561-6.

9 Holt RIG, Peveler RC, Byrne CD. Schizophrenia, the metabolic syndrome and diabetes. Diabet Med 2004; 21: 515-23.

10 Ananth J, Venkatesh R, Burgoyne K, Gunatilake S. Atypical antipsychotic drug use and diabetes. Psychother Psychosom 2002; 71: 244-54.

11 Erle G, Basso M, Federspil G, Sicolo N, Scandellari C. Effect of chlorpromazine on blood glucose and plasma insulin in man. Eur J Clin Pharmacol 1977; 11: 15-8.

12 Henderson D. Atypical antipsychotic-induced diabetes mellitus. How strong is the evidence? CNS Drugs 2002; 16: 77-89.

13 British Medical Association \& Royal Pharmaceutical Society of Great Britain. British National Formulary. BMJ Books \& Pharmaceutical Press, 2005.

14 Stroup DF, Berlin JA, Morton SC, Olkin I, Williamson GD, Rennie D, Moher D, Becker BJ, Sipe TA, Thacker SB. Meta-analysis of observational studies in epidemiology: a proposal for reporting. Meta-analysis Of Observational Studies in Epidemiology (MOOSE) group. JAMA 2000; 283: 2008-12.

15 STROBE. STROBE Statement. STrengthening the Reporting of OBservational studies in Epidemiology, 2006. (http://strobe-statement.org/).

16 American Diabetes Association. Report of the expert committee on the diagnosis and classification of diabetes mellitus. Diabetes Care 1997; 20 $1183-7$.

17 World Health Organization. Definition, Diagnosis and Classification of Diabetes Mellitus and its Complications: Part 1. Diagnosis and Classification of Diabetes Mellitus. World Health Organization, 1999.

18 Juni P, Altman DG, Egger M. Systematic Reviews in Health Care. Assessing the Quality of Randomized Controlled Trials. BMJ Books, 2001.

19 Woodward M. Epidemiology: Study Design and Data Analysis. (2nd edn) (ed. B. Raton). Chapman and Hall/CRC Press, 2005.

20 Lambert BL, Chou CH, Chang KY, Tafesse E, Carson W. Antipsychotic exposure and type 2 diabetes among patients with schizophrenia: a matched case-control study of California Medicaid claims. Pharmacoepidemiol Drug Saf 2005; 14: 417-25.

21 Lambert BL, Cunningham FE, Miller DR, Dalack GW, Hur K. Diabetes risk associated with use of olanzapine, quetiapine, and risperidone in veterans health administration patients with schizophrenia. Am J Epidemiol 2006; 164: 672-81.

22 Leslie DL, Rosenheck RA. Incidence of newly diagnosed diabetes attributable to first generation antipsychotic medications. Am J Psychiatry 2004; 161 1709-11.

23 Lund BC, Perry PJ, Brooks JM, Arndt S. Clozpine use in patients with schizophrenia and the risk of diabetes, hyperlipidemia, and hypertension. A claims-based approach. Arch Gen Psychiatry 2001; 58: 1172-6. 
24 Ollendorf DA, Joyce AT, Rucker M. Rate of new-onset diabetes among patients treated with first generation or conventional antipsychotic medications for schizophrenia. MedGenMed 2004; 6: 5 .

25 Zhao Z, Tunis SL, Loosbrock DL. Risk of diabetes for individuals with schizophrenia treated with antipsychotics. J Appl Res 2003; 3: 287-95.

26 Koro CE, Fedder DO, L'Italien GJ, Weiss SS, Magder LS, Kreyenbuhl J, Revicki DA, Buchanan RW. Assessment of independent effect of olanzapine and risperidone on risk of diabetes among patients with schizophrenia: population based nested case-control study. BMJ 2002; 325: 243-5.

27 Spitzer RL, Williams JBW, Gibbon M, First MB. Structured Clinical Interview for DSM-III-R (SCID). New York State Psychiatric Research Institute, 1987.

28 Chae B-J, Kang B-J. The effect of clozapine on blood glucose metabolism. Hum Psychopharmacol 2001; 16: 265-71.

29 Miller MJ, Molla PM. Prevalence of diabetes mellitus in patients receiving depot neuroleptics or clozapine. Arch Psychiatr Nurs 2005; 19: 30-4.

30 Cohen D, Dekker JJ, Peen J, Gispen-de Wied CC. Prevalence of diabetes mellitus in chronic schizophrenic inpatients in relation to long-term antipsychotic treatment. Eur Neuropsychopharmacol 2006; 16: 187-94.

31 World Health Organization. The ICD-10 Classification of Diseases and Related Health Problems. Tenth Revision. World Health Organization, 1992.

32 Kurt E, Oral ET, Verimli A. Comparison of the effects of classical and novel antipsychotic drugs on the insulin and glucose metabolisms of patients with schizophrenia or other psychotic disorders [Turkish]. Klinik Psikofarmakoloji Buelteni 2002; 12: 57-63.

33 Lindenmayer JP, Czobor P, Volavka J, Citrome L, Sheitman B, McEvoy JP, Cooper TB, Chakos M, Lieberman JA. Changes in glucose and cholesterol levels in patients with schizophrenia treated with second generation or first generation antipsychotics. Am J Psychiatry 2003; 160: 290-6.

34 Holt RIG, Peveler RC. Antipsychotic drugs and diabetes - an application of the Austin Bradford Hill criteria. Diabetologia 2006; 49: 1467-76.

35 Etminan M, Streiner DL, Rochon PA. Exploring the association between atypical neuroleptic agents and diabetes mellitus in older adults. Pharmacotherapy 2003; 23: 1411-5

36 Citrome L, Jaffe A, Levine J, Allingham B, Robinson J. Relationship between antipsychotic medication treatment and new cases of diabetes among psychiatric inpatients. Psychiatr Serv 2004; 55: 1006-13.

37 Gianfrancesco FD, Grogg AL, Mahmoud RA, Wang RH, Nasrallah HA. Differential effects of risperidone, olanzapine, clozapine, and conventional antipsychotics on type 2 diabetes: findings from a large health plan database. J Clin Psychiatry 2002; 63: 920-30.
38 Gianfresco F, White R, Wang R, Nasrallah HA. Antipsychotic-induced type 2 diabetes: evidence from a large health plan database. J Clin Psychopharmacol 2003; 23: 328-35.

39 Gupta S, Steinmeyer C, Frank B, Madhusoodanan S, Lockwood K, Lentz B, Keller P. Hyperglycemia and hypertriglyceridemia in real world patients on antipsychotic therapy. Am J Ther 2003; 10: 348-55.

40 Sacchetti E, Turrina C, Parrinello G, Brignoli O, Stefanini G, Mazzaglia G. Incidence of diabetes in a general practice population: a database cohort study on the relationship with haloperidol, olanzapine, risperidone or quetiapine exposure. Int Clin Psychopharmacol 2005; 20: 33-7.

41 Spanarello S, Beoni AM, Mina G, Colotto A. Antipsicotici e implicazioni metaboliche. Minerva Psychiatr 2002; 44: 39-45.

42 Brown RR, Estoup MW. Comparison of the metabolic effects observed in patients treated with ziprasidone versus olanzapine. Int Clin Psychopharmacol 2005; 20:105-12.

43 Caro JJ, Ward A, Levinton C, Robinson K. The risk of diabetes during olanzapine use compared with risperidone use: a retrospective database analysis. J Clin Psychiatry 2002; 63: 1135-9.

44 Melkersson KI, Dahl ML. Relationship between levels of insulin or triglycerides and serum concentrations of the atypical antipsychotics clozapine and olanzapine in patients on treatment with therapeutic doses. Psychopharmacol 2003; 170: 157-66.

45 Meyer JM. A retrospective comparison of weight, lipid, and glucose changes between risperidone- and olanzapine-treated inpatients: metabolic outcomes after 1 year. J Clin Psychiatry 2002; 63: 425-33.

46 Ananth, J, Parameswaran S, Gunatilake S. Side effects of atypical antipsychotic drugs. Curr Pharm Des 2004; 10: 2219-29.

47 Mackin P, Watkinson HM, Young AH. Prevalence of obesity, glucose homeostasis disorders and metabolic syndrome in psychiatric patients taking typical or atypical antipsychotic drugs: a cross-sectional study. Diabetologia 2005; 48: 215-21.

48 Jin H, Meyer JM, Jeste DV. Phenomenology of and risk factors for new-onset diabetes mellitus and diabetic ketoacidosis associated with atypical antipsychotics: an analysis of 45 published cases. Ann Clin Psychiatry 2002; 14: $59-64$.

49 Geddes J, Freemantle N, Harrison P, Bebbington P. Atypical antipsychotics in the treatment of schizophrenia: systematic review and meta-regression analysis. BMJ 2000; 321: 1371-6.

50 Subramaniam M, Chong SA, Pek E. Diabetes mellitus and impaired glucose tolerance in patients with schizophrenia. Can J Psychiatry 2003; 48: 345-7.

51 Hill A. The environment and disease: association or causation. Proc $R$ SOC Med 1965; 58: 295-330. 